\title{
Prenatal malnutrition and adult cognitive impairment: a natural experiment from the 1959-1961 Chinese famine
}

\author{
Ping $\mathrm{He}^{1,2,3}$, $\mathrm{Li} \mathrm{Liu}^{3}$, J. M. Ian Salas ${ }^{3}$, Chao Guo ${ }^{2,4}$, Yunfei Cheng ${ }^{2}$, Gong Chen ${ }^{2}$ and Xiaoying Zheng ${ }^{2,4 *}$ \\ ${ }^{1}$ China Center for Health Development Studies, Peking University, Beijing, 100191, People's Republic of China \\ ${ }^{2}$ Institute of Population Research, Peking University, Beijing, 100871, People's Republic of China \\ ${ }^{3}$ Bloomberg School of Public Health, Johns Hopkins University, Baltimore, MD 21205, USA \\ ${ }^{4}$ APEC Health Science Academy (HeSAY), Peking University, Beijing, 100871, People's Republic of China \\ (Submitted 28 June 2017 - Final revision received 2 January 2018 - Accepted 15 March 2018 - First published online 3 May 2018)
}

\section{Abstract}

The current measures of cognitive functioning in adulthood do not indicate a long-term association with prenatal exposure to the Dutch famine. However, whether such association emerges in China is poorly understood. We aimed to investigate the potential effect of prenatal exposure to the 1959-1961 Chinese famine on adult cognitive impairment. We obtained data from the Second National Sample Survey on Disability implemented in thirty-one provinces in 2006, and restricted our analysis to 387093 individuals born in 1956-1965. Cognitive impairment was defined as intelligence quotient (IQ) score under 70 and IQ of adults was evaluated by the Wechsler Adult Intelligence Scale China Revision. Famine severity was defined as excess death rate. The famine impact on adult cognitive impairment was estimated by difference-in-difference models, established by examining the variations of famine exposure across birth cohorts. Results show that compared with adults born in 1956-1958, those who were exposed to Chinese famine during gestation (born in 1959-1961) were at greater risk of cognitive impairment in the total sample. Stratified analyses showed that this effect was evident in males and females, but only in rural, not in urban areas. In conclusion, prenatal exposure to famine had an enduring deleterious effect on risk of cognitive impairment in rural adults.

Key words: Malnutrition: Famine: Cognitive impairment: Prenatal exposure

Early-life malnutrition affects the central nervous system of the human brain during gestation ${ }^{(1)}$ and has a long-term negative impact on cognitive functioning in later life ${ }^{(2)}$. A very limited number of longitudinal studies, conducted in Central America and the Caribbean nations including Barbados and Jamaica, showed that nutritional deprivation had severely deteriorated effect on cognitive impairment in adulthood ${ }^{(3,4)}$, and this functional loss can be compensated by early interventions ${ }^{(5)}$. Due to apparent ethical consideration, however, experimental studies regarding human cognitive impairment in adulthood after exposure to undernutrition in gestation almost do not exist ${ }^{(6)}$.

Famine, as a natural experiment, provides a unique opportunity to test the long-term association of prenatal malnutrition with adult cognitive functioning. In the past four decades, observational investigations on this issue were mainly conducted for the 1944-1945 Dutch famine and did not suggest a long-term effect of prenatal famine on adult cognitive functioning ${ }^{(6)}$. For example, the first study by Stein et al. ${ }^{(7)}$ in 1972 , found that prenatal exposure to famine was not related to prevalence rates of mild and severe mental retardation at about 19 years on male military recruits in the Netherlands. Two studies on the Dutch famine, afterwards, confirmed the findings using samples at ages between 56 and 59 years ${ }^{(8,9)}$.

Chinese famine is one of the most severe famines around the world, causing approximately 15-30 million excess deaths during 1959-1961 ${ }^{(10)}$. Nevertheless, compared with the Dutch famine, studies focusing on the association between the Chinese famine and cognitive functioning are limited partly due to data unavailability ${ }^{(11)}$. Among the few, most were at the regional level with small size ${ }^{(12)}$. In addition, analyses of the long-term association of prenatal famine exposure with adult cognitive impairment faced a number of methodological challenges, such as the identification of the causal effect of famine ${ }^{(10)}$. Previous studies, as a matter of fact, have not fully addressed these issues. One of the estimation methods to identify the causal effect of famine depends on the regional variation of famine across birth cohorts by using differencein-difference (DID) models ${ }^{(13)}$.

The National Sample Survey on Disability is a nationally representative data source that included measures of cognitive impairment in China. In this study, we attempted to analyse this population-based data to provide evidence on the long-term

Abbreviations: DID, difference-in-difference; EDR, excess death rate.

* Corresponding author: X. Zheng, fax +86 106275 1974; email xzheng@pku.edu.cn 
impact of prenatal exposure to the Chinese famine on cognitive impairment in adulthood on the basis of the identification strategy of DID. This study will contribute to the identification strategy of the long-term effect of famine on adult cognitive impairment and potential mechanisms for such effect.

The Chinese famine coincided with a nationwide movement, known as 'Great Leap Forward (GLF)', which started sweeping across China in 1958. The GLF aimed to bring about rapid industrialisation and overtake the level of Britain and the USA in a short time ${ }^{(10)}$. Contrary to expectation, however, the GLF severely disrupted agricultural production ${ }^{(14)}$. As a result, grain output dropped by $15 \%$ in 1959, and in the next 2 years, continued to drop to roughly $70 \%$ of the 1958 level $^{(15)}$. Meanwhile, the central government sharply increased grain procurement from the rural population. The plunge in grain output, excess procurement and severe weather disaster jointly caused a dramatic decline in energy intake, and the famine ensued in all regions of China ${ }^{(16)}$. From 1959 to 1961, death rates sharply increased while fertility rates rapidly decreased at the same time ${ }^{(16)}$. By 1962, both death and birth rates returned to a normal level ${ }^{(17)}$.

\section{Methods}

\section{Study participants}

We obtained data from the Second National Sample Survey on Disability implemented in thirty-one provinces in 2006. The aim of the survey was to investigate the prevalence, causes and severity of disabilities, as well as the living conditions and health service needs of the disabled. Multistage stratified random cluster sampling, with probability proportional to size, was used in 734 counties (districts), 2980 towns (streets) and 5964 communities (villages) from all provinces, autonomous regions and municipalities. More details of sampling were presented in our previous study ${ }^{(18)}$. The survey sample size was 2526145 , accounting for $1.9 / 1000$ inhabitants of China ${ }^{(19)}$.

In this study, the term 'prenatal famine' refers to maternal exposure to famine during the roughly $300 \mathrm{~d}$ from the periconception to delivery ${ }^{(20)}$. We restricted our analysis to 1956 1965 birth cohorts, mainly because of avoiding other natural disasters before and after the famine, including the extremely cold weather of 1954-1955 and the Chinese Cultural Revolution of 1966-1976 $6^{(13)}$. We defined adults born in 1959-1962 as those with prenatal exposure to famine, while those born in 19561958 and 1963-1965 were not prenatally exposed. In total, we selected a subsample of 387093 adults who were born between 1956 and 1965, at the ages of 41-50 years during the survey time.

\section{Ethics approval}

The survey was conducted in thirty-one provinces by the Leading Group of the National Sample Survey on Disability and the National Bureau of Statistics. The survey was approved by the China State Council (no. 20051104) and implemented within the legal framework governed by the Statistical Law of the People's Republic of China (1996 Amendment). All respondents provided consent to the Chinese government, which covered their participation in the survey and the clinical assessment process.

\section{Measures}

Cognitive impairment. The outcome variable was whether or not an individual had cognitive impairment. Individuals with cognitive impairment were ascertained by the combination of questionnaire screening and medical diagnosis by psychiatrists. Cognitive impairment was defined as intelligence quotient (IQ) $<70$ and the age of onset before 18 years ${ }^{(21)}$. The IQ for adults was evaluated by the Wechsler Adult Intelligence Scale - China Revision, which has been widely utilised in China ${ }^{(22)}$. Survey interviewers were recruited from local primary care institutions and screened adults using screening questionnaires in households. The interviewers were trained by the provincial expert teams on survey methods and participant screening. The screening questions included: (1) do you or your family members have difficulty in learning; and (2) does anybody in your family have poor life or workability and need help from others? If the screeners found that the subjects had potential cognitive impairment, they were referred to psychiatrists for medical diagnosis of cognitive impairment.

Famine severity. The uniqueness of the Chinese famine not only rests on its long duration and extreme severity but also on its substantial variation in severity across regions ${ }^{(10)}$. This regional variation in famine severity, combined with differences in cognitive impairment, provides a critical opportunity to identify the causal effects of famine.

Following previous studies ${ }^{(10,23)}$, we used the excess death rate (EDR) as a proxy of famine severity at the province level. The EDR is calculated as the gap between the average death rate during the famine (1959-1961) and 3 years before the famine (1956-1958). The death data across provinces were obtained from the previous studies ${ }^{(16,24)}$. According to the administrative region division during 1956-1965, Hainan and Chongqing were included in Guangdong and Sichuan province, respectively. We dropped Tibet due to lack of population data during the famine period. As a result, we obtained the EDR values of twenty-eight provinces, ranging from 0.07 to 28.63/ 1000 persons with a mean of $6 \cdot 94$, representing that the death rate during the famine period was $0.694 \%$ higher than the mean death rate in the 3 years before the famine in these provinces.

In addition, we employed the cohort size shrinkage index $(\mathrm{CSSI})^{(25,26)}$, measured by comparing the size of the famine cohorts relative to the surrounding non-famine cohorts in the population, as an alternative measure of famine severity to check the robustness of regressions. The details of CSSI have been shown elsewhere ${ }^{(13,27)}$.

Control variables. Control variables in this study included sex (male $v$. female) and residency (urban $v$. rural). We defined residency based on current living address at the individual level.

\section{Analytic approach}

The famine impact on adult cognitive impairment was estimated by the DID models, which examined the regional 
variations of famine exposure across birth cohorts. The idea behind this approach is that we can use the birth year to identify whether or not an individual was prenatally exposed during the famine. Meanwhile, we can also rely on the famine severity across regions to identify the variation of famine exposure in the same birth cohorts. The detailed rationale of using this method to infer the causal effect of famine was explained elsewhere ${ }^{(10)}$ and was broadly used in Chinese famine studies $^{(13,23,25,28)}$. Logit regression models with the DID estimators were fitted as the following:

$Y_{i j k}=B_{0}+\delta \mathrm{EDR}_{j}+\varphi_{k}$ Cohort $_{k}+\sum_{k=1956}^{1965} \beta_{k}\left(\mathrm{EDR}_{r} \times \operatorname{Cohort}_{k}\right)+V X_{i j k}$,

where $Y_{i j k}$ is the risk of cognitive impairment for individual $i$, born in province $j$ and year $k$ ( $k$ ranges from 1956 to 1965), $\mathrm{EDR}_{j}$ is the EDR of province $j, \varphi_{k}$ represents the cohort fixed effect, $X_{i j k}$ constitutes a vector of control variables involving sex and residency, and $V$ is the scalar that contains the corresponding coefficients of the covariates. Standard errors are clustered at the province level to deal with potential heteroscedasticity and serial correlation problems ${ }^{(29)}$.

The coefficient of the interaction between the EDR and the birth cohort dummies, namely $\beta_{k}$, evaluates the impact of the prenatal exposure to famine on the risk of cognitive impairment in the DID models. The estimates of this effect using the interaction term in non-linear DID models were shown elsewhere $^{(30,31)}$. To estimate the average effect across provinces, we multiplied the interaction coefficient by 6.94 , the mean of EDR in all provinces.

For all models, we first analysed all samples and subsamples by residency and sex. A $P$ value of $<0.05$ was considered statistically significant. The software Stata version 13 for Windows (StataCorp) was used for the statistical analysis.

\section{Results}

Table 1 shows the characteristics of the sample by birth cohorts. Overall, of the 387093 adults, $38.24 \%$ lived in the urban area, $49.78 \%$ were female and $0.61 \%$ had cognitive impairment. The proportion of urban population accounting for the total population fluctuated before, during and after famine period, peaking at $42.21 \%$ in 1960 . The proportion of females was stable from 1956 to 1965, representing about a half of the total population.

Table 2 presents the odds ratios for risk of cognitive impairment predicted by famine exposure. In the total sample, adults born in 1959, 1960 and 1961 were $1 \cdot 11$ (95\% CI 1.02, 1.20), 1.20 (95\% CI 1.07, 1.35) and 1.21 (95\% CI 1.02, 1.43) times more likely to have cognitive impairment than those who were born between 1956 and 1958, respectively, after adjusting for sex and residency.

Table 3 shows the odds ratios for risk of cognitive impairment predicted by famine exposure by urban and rural residency. In the rural sample, compared with pre-famine cohorts, adults born in 1960 had a higher rate of cognitive impairment, with an OR of 1.20 (95\% CI 1.03, 1.40). In the urban sample, there was no statistically significant difference in the prevalence of cognitive impairment between famine cohorts and pre-famine cohorts.

Table 4 illustrates the odds ratios for cognitive impairment of famine cohorts in relative to pre-famine cohorts by sex. In the male population, those who were born in 1959 were more likely than their pre-famine peers to have cognitive impairment, with an OR of 1.18 (95\% CI 1.06, 1.32). In the female population, individuals born in 1960 and 1961 had higher rates of cognitive impairment than those in pre-famine cohorts, with an OR of 1.37 (95\% CI $1.15,1.63)$ and 1.32 (95\% CI $1.12,1.55)$, respectively.

The robustness analyses suggested that when the post-famine cohorts (born in 1963-1965) were used as the reference group, we could still observe a higher rate of cognitive impairment among adults born in 1961 (online Supplementary Table S1). In addition, when we used the CSSI as an alternative measure of

Table 2. Risk of cognitive impairment predicted by famine exposure, based on logit regression with difference-in-difference estimator ( $n$ 235697) (Odds ratios and $95 \%$ confidence intervals)

\begin{tabular}{llll}
\hline Birth cohorts & OR $^{\star}$ & $95 \% \mathrm{Cl}$ & $P+$ \\
\hline 1959 & 1.11 & $1.02,1.20$ & 0.017 \\
1960 & 1.20 & $1.07,1.35$ & 0.002 \\
1961 & 1.21 & $1.02,1.43$ & 0.030 \\
1962 & 1.03 & $0.91,1.18$ & 0.617 \\
Pre-famine cohorts (1956-1958) & Ref. & & \\
\hline
\end{tabular}

Ref., referent value.

* Adjusting for sex and urban-rural residence.

$\dagger P$ value is statistically significant at the 0.05 level.

Table 1. Characteristics of sample, by birth cohorts

\begin{tabular}{|c|c|c|c|c|c|}
\hline Birth cohorts & Year & Sample size $(n)$ & Urban (\%) & Female (\%) & Prevalence of cognitive impairment (\%) \\
\hline \multirow[t]{3}{*}{ Pre-famine cohorts (1956-1958) } & 1956 & 38794 & $35 \cdot 66$ & $49 \cdot 71$ & 0.60 \\
\hline & 1957 & 39004 & $36 \cdot 57$ & $49 \cdot 73$ & 0.67 \\
\hline & 1958 & 41278 & $37 \cdot 60$ & $49 \cdot 27$ & 0.64 \\
\hline \multirow[t]{4}{*}{ Famine cohorts (1959-1962) } & 1959 & 33666 & 38.99 & $49 \cdot 62$ & 0.65 \\
\hline & 1960 & 28964 & $42 \cdot 21$ & $49 \cdot 90$ & 0.53 \\
\hline & 1961 & 26579 & 42.09 & 49.86 & 0.63 \\
\hline & 1962 & 27412 & 39.02 & $50 \cdot 16$ & 0.62 \\
\hline \multirow[t]{3}{*}{ Post-famine cohorts (1963-1965) } & 1963 & 50495 & $38 \cdot 14$ & $49 \cdot 45$ & 0.52 \\
\hline & 1964 & 53921 & 38.44 & $49 \cdot 98$ & 0.59 \\
\hline & 1965 & 46980 & $36 \cdot 61$ & $50 \cdot 21$ & 0.64 \\
\hline All cohorts (1956-1965) & $1956-1965$ & 387093 & $38 \cdot 24$ & $49 \cdot 78$ & 0.61 \\
\hline
\end{tabular}


Table 3. Risk of cognitive impairment predicted by famine exposure, based on logit regression with difference-in-difference estimator, by urban-rural residency

(Odds ratios and $95 \%$ confidence intervals)

\begin{tabular}{|c|c|c|c|c|c|c|}
\hline \multirow[b]{2}{*}{ Birth cohorts } & \multicolumn{3}{|c|}{ Rural ( $n$ 144 842) } & \multicolumn{3}{|c|}{ Urban ( $n 90855)$} \\
\hline & $\mathrm{OR}^{*}$ & $95 \% \mathrm{Cl}$ & $P \dagger$ & $\mathrm{OR}^{*}$ & $95 \% \mathrm{Cl}$ & $P \dagger$ \\
\hline 1959 & $1 \cdot 10$ & $0.98,1.23$ & 0.114 & $1 \cdot 11$ & $0.87,1.41$ & 0.392 \\
\hline 1960 & 1.20 & $1.03,1.40$ & 0.020 & 1.20 & $0.99,1.45$ & 0.057 \\
\hline 1961 & 1.22 & $0.97,1.54$ & 0.083 & 1.13 & $0.96,1.34$ & 0.144 \\
\hline 1962 & $1 \cdot 10$ & $0.94,1.28$ & 0.235 & 0.77 & $0.39,1.50$ & 0.441 \\
\hline $\begin{array}{l}\text { Pre-famine cohorts } \\
\qquad(1956-1958)\end{array}$ & Ref. & & & Ref. & & \\
\hline
\end{tabular}

Ref., referent values.

* Adjusting for sex.

$\dagger P$ value is statistically significant at the 0.05 level.

Table 4. Risk of cognitive impairment predicted by famine exposure, based on logit regression with difference-in-difference estimator, by sex (Odds ratios and $95 \%$ confidence intervals)

\begin{tabular}{|c|c|c|c|c|c|c|}
\hline \multirow[b]{2}{*}{ Birth cohorts } & \multicolumn{3}{|c|}{ Male ( $n$ 118516) } & \multicolumn{3}{|c|}{ Female $(n 117181)$} \\
\hline & $\mathrm{OR}^{*}$ & $95 \% \mathrm{Cl}$ & $P+$ & $\mathrm{OR}^{*}$ & $95 \% \mathrm{Cl}$ & $P \dagger$ \\
\hline 1959 & $1 \cdot 18$ & $1.06,1.32$ & 0.002 & 0.97 & $0.86,1.10$ & 0.627 \\
\hline 1960 & 1.01 & $0.86,1.19$ & 0.858 & 1.37 & $1.15,1.63$ & $<0.001$ \\
\hline 1961 & 1.06 & $0.86,1.30$ & 0.600 & 1.32 & $1 \cdot 12,1.55$ & 0.001 \\
\hline 1962 & 0.97 & $0.85,1.11$ & 0.694 & $1 \cdot 10$ & $0.91,1.32$ & 0.318 \\
\hline $\begin{array}{l}\text { Pre-famine cohorts } \\
\qquad(1956-1958)\end{array}$ & Ref. & & & Ref. & & \\
\hline
\end{tabular}

Ref., referent values.

* Adjusting for urban-rural residency.

$\dagger P$ value is statistically significant at the 0.05 level.

famine severity, we found that the results were similar (online Supplementary Table S2).

\section{Discussion}

We investigated whether prenatal exposure to the Chinese famine would predict cognitive impairment in adulthood. We found that prenatal exposure to famine had an enduring deleterious effect on cognitive impairment in adulthood. This effect was evident in both males and females, in rural but not in urban areas.

The effect of famine exposure found in this study indicates that prenatal malnutrition has a long-term negative effect on cognitive impairment in adulthood. This result is in line with previous studies in China. For instance, Chinese studies suggested a long-term association between prenatal famine exposure and adulthood cognitive ability, as defined by memory, calculation or word test ${ }^{(11,12,32)}$. In addition, two longitudinal investigations suggested that in low-resource countries, prenatal nutritional deprivation has a negative impact on human cognitive function in adulthood ${ }^{(3,4)}$. This study further verified the Chinese famine effect on cognitive impairment by examining famine survivors aged 41-50 years from the perspective of a natural experiment. Furthermore, laboratory studies in animals revealed the potential mechanisms on how prenatal exposure to malnutrition impacted on adult cognitive impairment. For example, studies on rats showed that prenatal undernutrition was associated with the alteration of brain development ${ }^{(33)}$, and then had a negative impact on cognitive function in later life ${ }^{(34,35)}$. As well, animal studies showed that dietary deprivation in utero influenced sub-regions of the hippocampus ${ }^{(35)}$, and prenatal malnutrition on protein changed the response of medial prefrontal neurons to stress ${ }^{(36)}$.

The negative impact of Chinese prenatal famine on cognitive impairment observed in this study differs from that of the Dutch famine. An excellent review concluded that there was no longterm association between prenatal exposure to the Dutch famine and cognitive functioning in adulthood ${ }^{(6)}$, on the basis of three studies ${ }^{(7-9)}$. We cannot directly compare our study with the Dutch studies because the effect of prenatal malnutrition on adult health is determined by multiple factors ${ }^{(13)}$. For example, the Dutch and Chinese famines were very different. The Dutch famine emerged in a Western, rich country, and mainly impacted urban residents in selected places for less than half a year ${ }^{(20)}$. By contrast, the Chinese famine occurred in an eastern, poor country, and primarily affected rural people across the entire country for about 3 years ${ }^{(37)}$. Clearly, the Chinese famine could have induced more chronic malnutrition among the Chinese population. Furthermore, studies of the two famines vary in the study design and data availability. The Dutch famine contained complete vital statistics and detailed nutrition intake before, during and after famine so that exposure timing and duration can be precisely identified for each individual. However, it is almost impossible to obtain vital statistics, especially at the county level, for the Chinese famine studies ${ }^{(20)}$; instead, most studies used birth cohort as a proxy of famine exposure and defined mortality or fertility indicators as the intensity of exposure at the province level ${ }^{(10,11)}$

We only found the long-term impact of prenatal famine exposure on adult cognitive impairment in rural areas. This is likely because famine severity was much greater in rural China $^{(28)}$. The similar rural-urban differentials have been observed for other health outcomes like schizophrenia ${ }^{(38)}$. The disparity of famine between urban and rural areas may be in part explained by the registration system, namely 'Hukou' in China, as well as the system of grain procurement from the rural population at that time ${ }^{(11)}$. The strict registration system of Hukou was launched in 1951, and furtherly reinforced by the end of the 1950s. Chinese residents, regulated by the Hukou system, were prohibited from free migration, especially from rural to urban areas ${ }^{(39)}$. More importantly, in the famine years, rural families were forced to turn in a larger amount of grain despite a huge shrinkage of food production, and consequently caused more severe starvation in rural China; by contrast, urban residents had the legal rights to receive a certain amount of food from state grain store during the famine, and therefore experienced a relatively smaller impact of the famine ${ }^{(38)}$.

This study is subject to several limitations. Like other famine studies in China, we need to be cautious to interpret our findings. The first potential threat to the validity of our finding comes from famine-associated selected mortality. It is well understood that those who were more severely affected by the famine were more likely to have died in the famine. Meanwhile, the famine survivors with more serious conditions were also less likely to have survived to middle age by the survey time ${ }^{(11)}$. 
In the absence of mortality data associated with cognitive impairment, we were unable to correct the underlying bias, but this would likely underestimate rather than overestimate famine effect. The second source of potential bias is population migration. This study speculated birth place according to individuals' current living place at the province level, which may lead to the imprecise identification of family severity across provinces. However, the Hukou (passport) system, initiated in the 1950s, greatly restricted population migration in China, especially from rural to urban areas ${ }^{(40)}$. In fact, during the period of famine, interprovincial migration only constituted less than $1 \%$ of the total population ${ }^{(10)}$. In addition, compared to the Dutch famine with high-quality birth and energetic intake statistics across the life course, we acknowledge that there are some limitations in the measurement of the severity of Chinese famine. But so far, within the scope of the existing data, the EDR and CSSI used in this study are two reasonable measures to assess the intensity of the Chinese famine. Finally, the screening process may cause that cognitive impairment data were more likely to be obtained from respondents who were willing to selfidentify or to identify family members as having a potential cognitive impairment. Due to lack of information on whether or not an individual got measured for cognitive impairment, we could not run a covariate balance test to see if there was possible sample selection. Despite these limitations, our study extends the long-term impact of the Chinese famine from cognitive ability to cognitive impairment based on a large-scale, nationally representative survey.

\section{Conclusions}

We found that prenatal famine has a long-term deteriorate impact on cognitive impairment in adulthood, and the impact was evident among both females and males in rural areas. Further studies need to explore the difference between the Chinese and Dutch famine and the corresponding mechanism on this issue. Policies or programmes should be developed to intervene in those who were exposed to prenatal malnutrition to address their cognitive impairments.

\section{Acknowledgements}

The authors thank the provincial and municipal federations of disabled people for their support in data collection and management.

The work was supported by the Key National Project (973) of Study on the Mechanisms of Interaction between Environment and Genetics of Birth Defects in China (grant no. 2007CB5119001), the Key State funds for social science project (Research on Disability Prevention Measurement in China, grant no. 09\&ZD072) and the State Scholarship Fund (grant no. 201606010254).

P. H. initiated the study, analysed data and wrote the original article. L. L. gave critical suggestions on data analysis and revised the paper. J. M. I. S., C. G., Y. C. and G. C. provided advice on revising the paper. X. Z. supervised all aspects of implementation of the paper and contributed to writing the article. All authors critically interpreted the findings and edited the article.

The authors declare that there are no conflicts of interest.

\section{Supplementary material}

For supplementary material/s referred to in this article, please visit https://doi.org/10.1017/S0007114518000958

\section{References}

1. Gillette-Guyonnet S \& Vellas B (2008) Caloric restriction and brain function. Curr Opin Clin Nutr Metab Care 11, 686-692.

2. Harding JE (2001) The nutritional basis of the fetal origins of adult disease. Int J Epidemiol 30, 15-23.

3. Galler JR, Bryce C, Waber DP, et al. (2012) Socioeconomic outcomes in adults malnourished in the first year of life: a 40-year study. Pediatrics 130, e1-e7.

4. Waber DP, Bryce CP, Girard JM, et al. (2014) Impaired IQ and academic skills in adults who experienced moderate to severe infantile malnutrition: a 40-year study. Nutr Neurosci 17, 58-64.

5. Walker SP, Chang SM, Vera-Hernandez M, et al. (2011) Early childhood stimulation benefits adult competence and reduces violent behavior. Pediatrics 127, 849-857.

6. Lumey LH, Stein AD \& Susser E (2011) Prenatal famine and adult health. Annu Rev Public Health 32, 237-262.

7. Stein Z, Susser M, Saenger G, et al. (1972) Nutrition and mental performance. Science 178, 708-713.

8. de Groot RH, Stein AD, Jolles J, et al. (2011) Prenatal famine exposure and cognition at age 59 years. Int J Epidemiol $\mathbf{4 0}$, 327-337.

9. de Rooij SR, Wouters H, Yonker JE, et al. (2010) Prenatal undernutrition and cognitive function in late adulthood. Proc Natl Acad Sci U S A 107, 16881-16886.

10. Chen Y \& Zhou LA (2007) The long-term health and economic consequences of the 1959-1961 famine in China. J Health Econ 26, 659-681.

11. Kim S, Fleisher B \& Sun JY (2016) The long-term health effects of fetal malnutrition: evidence from the 1959-1961 China Great Leap Forward Famine. Health Econ 26, 1264-1277.

12. Wang C, An Y, Yu H, et al. (2016) Association between exposure to the Chinese famine in different stages of early life and decline in cognitive functioning in adulthood. Front Behav Neurosci 10, 146.

13. Huang C, Phillips MR, Zhang Y, et al. (2013) Malnutrition in early life and adult mental health: evidence from a natural experiment. Soc Sci Med 97, 259-266.

14. Kim S, Deng Q, Fleisher BM, et al. (2014) The lasting impact of parental early life malnutrition on their offspring: evidence from the China Great Leap Forward Famine. World Dev 54, 232-242.

15. Li W \& Yang DT (2005) The Great Leap Forward: anatomy of a central planning disaster. J Political Econ 113, 840-877.

16. Lin JY \& Yang DT (2000) Food availability, entitlements and the Chinese famine of 1959-61. Econ J 110, 136-158.

17. Almond D, Edlund L, Li H, et al. (2007) Long-term effects of the 1959-1961 China famine: mainland China and Hong Kong. NBER Working Paper Series. Cambridge, MA. http://www.nber.org/ papers/w13384 (accessed March 2017).

18. Zheng X, Chen G, Song X, et al. (2011) Twenty-year trends in the prevalence of disability in China. Bull World Health Organ 89, 788-797.

19. He P, Chen G, Wang Z, et al. (2017) Children with motor impairment related to cerebral palsy: prevalence, severity and concurrent impairments in China. J Paediatr Child Health $\mathbf{5 3}$, 480-484.

20. Susser E St \& Clair D (2013) Prenatal famine and adult mental illness: interpreting concordant and discordant results from the Dutch and Chinese Famines. Soc Sci Med 97, 325-330.

21. Wu L, Qiu Z, Wong D, et al. (2010) The research on the status, rehabilitation, education, vocational development, social 
integration and support services related to intellectual disability in China. Res Dev Disabil 31, 1216-1222.

22. Xiong NN, Ye Q, Zhang S, et al. (2009) Investigation on prevalence and causes of children with intellectual disability in China. Chinese J Child Health Care 17, 48-50.

23. Meng X, Qian N \& Yared P (2015) The institutional causes of China's Great Famine, 1959-1961. Rev Econ Stud 82, 1568-1611.

24. Peng X (1987) Demographic consequences of the Great Leap Forward in China's Provinces. Popul Dev Rev 13, 639-670.

25. Huang C, Guo C, Nichols C, et al. (2014) Elevated levels of protein in urine in adulthood after exposure to the Chinese famine of 1959-61 during gestation and the early postnatal period. Int J Epidemiol 43, 1806-1814.

26. Huang C, Li Z, Wang M, et al. (2010) Early life exposure to the 1959-1961 Chinese famine has long-term health consequences. J Nutr 140, 1874-1878.

27. Huang C, Li Z, Narayan KV, et al. (2010) Bigger babies born to women survivors of the 1959-1961 Chinese famine: a puzzle due to survival selection? J Dev Orig Health Dis 1, 412-418.

28. Hu XF, Liu GG \& Fan M (2016) Long-term effects of famine on chronic diseases: evidence from China's Great Leap Forward Famine. Health Econ 26, 922-936.

29. Bertrand M, Duflo E \& Mullainathan S (2004) How much should we trust differences-in-differences estimates? QJ Econ 119, 249-275.

30. Athey S \& Imbens GW (2006) Identification and inference in nonlinear difference-in-differences models. Econometrica $\mathbf{7 4}$, 431-497.

31. Puhani PA \& Sonderhof K (2010) The effects of a sick pay reform on absence and on health-related outcomes. J Health Econ 29, 285-302.
32. Tan CM, Zhibo $T$ \& Zhang $X$ (2014) Sins of the fathers: the intergenerational legacy of the 1959-1961 Great Chinese Famine on children's cognitive development. IFPRI Discussion Paper 1351. Washington, DC. https://ideas.repec.org/p/ fpr/ifprid/1351.html (accessed March 2017)

33. Bedi KS (2003) Nutritional effects on neuron numbers. Nutr Neurosci 6, 141-152.

34. Duran P, Cintra L, Galler J, et al. (2005) Prenatal protein malnutrition induces a phase shift advance of the spontaneous locomotor rhythm and alters the rest/activity ratio in adult rats. Nutr Neurosci 8, 167-172.

35. Ranade SC, Rose A, Rao M, et al. (2008) Different types of nutritional deficiencies affect different domains of spatial memory function checked in a radial arm maze. Neuroscience 152, 859-866.

36. Rosene DL, Lister JP, Schwagerl AL, et al. (2004) Prenatal protein malnutrition in rats alters the c-Fos response of neurons in the anterior cingulate and medial prefrontal region to behavioral stress. Nutr Neurosci 7, 281-289.

37. Lin JY \& Yang DT (2000) Food availability, entitlements and the Chinese famine of 1959-61. Econ J 110, 136-158.

38. Xu MQ, Sun WS, Liu BX, et al. (2009) Prenatal malnutrition and adult schizophrenia: further evidence from the 1959-1961 Chinese famine. Schizophr Bull 35, 568-576.

39. Wang F-L (2005) Organizing Through Division and Exclusion: China's Hukou System. San Francisco, CA: Stanford University Press.

40. Gooch E (2017) Estimating the long-term impact of the Great Chinese Famine (1959-61) on Modern China. World Dev 89, 140-151. 